\title{
Aberrant crypt foci: endoscopic assessment and cell kinetics characterization
}

\author{
Pedro Figueiredo • Maria Donato • Marta Urbano • \\ Helena Goulão • Hermano Gouveia • Carlos Sofia • \\ Maximino Leitão • Diniz Freitas
}

Accepted: 15 August 2008

(C) Springer-Verlag 2008

\begin{abstract}
Background and aims Aberrant crypt foci (ACF) are preneoplastic lesions in animal models of colorectal cancer. The aim of the study is to investigate if ACF are involved in human colorectal carcinogenic process and if they can be
\end{abstract}

P. Figueiredo $\cdot$ H. Gouveia $\cdot$ C. Sofia $\cdot$ M. Leitão $\cdot$ D. Freitas Department of Gastroenterology,

Hospitais da Universidade de Coimbra,

Avenida Bissaya Barreto,

3000-075 Coimbra, Portugal

H. Gouveia

e-mail: hermanogouveia@netcabo.pt

C. Sofia

e-mail: carlossofia@huc.min-saude.pt

M. Leitão

e-mail: maximino.leitao@clix.pt

D. Freitas

e-mail: gastro@huc.min-saude.pt

M. Donato $\cdot$ M. Urbano $\cdot$ H. Goulão

Centro de Gastrenterologia,

University of Coimbra-Medical School,

Rua Larga,

3004-504 Coimbra, Portugal

M. Donato

e-mail: gastro@ci.uc.pt

M. Urbano

e-mail: marta.urbano@netcabo.pt

H. Goulão

e-mail: gastro@huc.min-saude.pt

P. Figueiredo $(\bowtie)$

Serviço de Gastrenterologia,

Hospitais da Universidade de Coimbra,

Avenida Bissaya Barreto,

3000-075 Coimbra, Portugal

e-mail: pedro.n.figueiredo@clix.pt helpful in predicting the presence of a colorectal neoplasia. Methods The study included, between 2003 and 2005, 182 patients, 62 with adenoma, 55 with colorectal carcinoma, 53 without colorectal lesions, and 12 with nonneoplastic mucosal polyps. The number of rectal ACF was determined by colonoscopy. Proliferation and apoptosis indexes were evaluated by immunohistochemistry in rectal ACF, in normal rectal mucosa, and in carcinomatous tissue.

Results The mean number of rectal ACF in patients with rectal neoplasia was 12.64 , significantly higher than in patients with neoplastic lesions elsewhere in the colon $(p=$ 0.01). The apoptosis index in ACF of patients with colorectal carcinoma or adenoma aged 50 years or older was significantly lower than in younger patients $(1.3 \%$ vs $2.7 \%, p=0.01)$ and, in patients with carcinoma, lower than in normal mucosa $(1.1 \%$ vs $2.1 \%, p=0.002)$. The proliferation index was significantly higher in ACF of patients with colorectal neoplasia aged less than 50 years than in normal mucosa $(10.9 \%$ vs $7.7 \%, p=0.02)$. The apoptosis index in ACF foci of patients with carcinoma (1.1\%) was significantly lower than in patients without lesions $(2.2 \%)$ and than in normal mucosa $(2 \%)$. The mean number of ACF is significantly higher in patients with polyps larger than $1 \mathrm{~cm}(11.28$ vs $6.27, p=0.02)$. Conclusion Aberrant crypt foci probably precede the appearance of neoplasia and may be helpful in predicting the presence of a colorectal neoplastic lesion.

Keywords Colorectal cancer - Aberrant crypt foci . Cell kinetics · Colonoscopy · Magnification · Chromoscopy

\section{Introduction}

Colorectal carcinoma (CRC) is one of the most commonly diagnosed cancers, with a million new cases registered in 
2002 [1]. It is a lesion that can be prevented by adequate screening measures, of which colonoscopy is the standard method [2].

The carcinogenic pathway involves the accumulation of genetic mutations in normal mucosa, determining phenotypic modifications with biological and morphologic consequences [3]. In this context, it is relevant to study the cellular kinetics in the apparently normal colonic mucosa of patients with $\mathrm{CRC}$, as well as in individuals that present an increased risk of CRC. Data about proliferation in normal colonic mucosa of patients with adenomatous polyps are contradictory; papers exist that show a higher proliferation rate than in patients without colonic lesion [4-7], while others demonstrate the contrary [8]. There seems to be no correlation between the proliferation index in normal mucosa and the capacity of predicting a neoplastic lesion in the future $[6,8]$. When considering apoptosis, this is known to be significantly less in the normal mucosa of patients with adenoma than in controls $[9,10]$, and the proapoptotic response induced by biliary acids in the normal mucosa is significantly smaller in patients with a history of CRC than in individuals who are neoplasia free [11]. These data about cellular kinetics in normal mucosa should be interpreted cautiously because we have to consider that supposedly "normal" mucosa is, in reality, a heterogeneous entity. In fact, the known existence of colonic and rectal aberrant crypt foci (ACF), variable in number and histological nature, suggests that some of the results reported in the literature on cellular kinetics may be influenced by their presence in the biopsies selected for study.

Bird first described ACF in methylene-blue stained whole mount preparations of rodent colon treated with a colon carcinogen (azoxymethane) [12]. They are defined as a group of one or more crypts that are larger than most crypts in the field and have a thickened layer of epithelial cells that stain more intensely with methylene-blue; they often have a slit-shaped luminal opening, an increased pericryptal space, and are elevated from the focal plane of the microscope [13]. The assumption that ACF are biomarkers of CRC has resulted from animal studies, which show that colon carcinogens induce the appearance of ACF [14] in a dose-related number [15]. The modulation of ACF by chemopreventive agents with proven activity in CRC is also described in animal studies [16-18]. With the emergence of magnification and chromoscopy, it is now possible to perform endoscopic in vivo human studies [1926]. Although there are a number of arguments supporting the preneoplastic nature of ACF, such as an average number that increases with age [23], a higher prevalence in patients with neoplastic lesion of the colon [23], the identification of $\mathrm{ACF}$ with carcinoma in situ in a patient with sporadic colon cancer [27], the presence of ACF with dysplasia and APC mutations in patients with familial adenomatous polyposis $[28,29]$, and the occurrence of microsatellite instability in
ACF picked from patients with Lynch syndrome [30], the truth is that doubt still persists with regard the preneoplastic nature of ACF, which is unquestionable only in animal models of intestinal cancer.

The aim of this study is to investigate whether ACF can be considered precursors of the adenoma carcinoma sequence. For this purpose, they were studied alongside known CRC risk factors, in the vicinity of neoplastic lesions in order to find out if their characteristics vary in accordance with the distance to the colorectal neoplasia and compared with normal colorectal mucosa and carcinomatous tissue. The possibility that rectal ACF could predict the presence of a colorectal neoplasia, maybe even contributing to the stratification of the CRC risk, was also evaluated by studying ACF characteristics in several groups of patients with and without adenomas or carcinomas, as well as in individuals without colorectal lesions.

\section{Material and methods}

The study included patients referred for colonoscopy at the Department of Gastroenterology of the Coimbra University Hospitals between January 2003 and July 2005. A sample of 182 patients was evaluated, consisting of 106 males (58.2\%) and 76 females, with a mean age of 61.8 years and a range of 23-89 years.

Inclusion criteria were the presence of colorectal carcinoma, adenoma, or nonneoplastic mucosal colorectal polyp, as well as patients without colorectal lesions. The exclusion criteria were: pregnancy, age under 18 years, personal or family history of familial adenomatous polyposis or of any other hereditary gastrointestinal polyposis syndrome, Lynch Syndrome, inflammatory bowel disease, previous intestinal resection, history of malignant neoplasia submitted to radiotherapy or chemotherapy, hemorrhagic diathesis, severe hepatic, cardiac or renal illness, use of nonsteroid anti-inflammatory drugs, calcium, selenium, folate, hormonal replacement therapy or ursodeoxycholic acid in the preceding 3 months, and inclusion in postpolypectomy colonoscopic surveillance protocol.

After giving signed informed consent, the patients were subjected to an initial clinical evaluation to assess personal and family history of colorectal neoplasia and alcohol and tobacco consumption. With regard to family history of colorectal cancer or adenomatous polyps, the patients were classified into three classes according to criteria proposed by Winawer et al. [31]: a first-degree relative with colon cancer or adenomatous polyps diagnosed at age under 60 years or two first-degree relatives diagnosed with colorectal cancer at any age (class 2), a first-degree relative with colon cancer or adenomatous polyp diagnosed at age over 60 years or two second-degree relatives with colorectal 
cancer (class 1), and absence of increased risk (class 0; Table 2). Alcohol consumption habits were expressed in grams of alcohol per day. In accordance with published data $[32,33]$, the patients were classified into three groups: daily intake of over $30 \mathrm{~g}$ of alcohol; daily intake of less than $30 \mathrm{~g}$ of alcohol, and abstinence (when this exceeded 10 years). Following the data from the literature [34], patients were also classified according to the average number of cigarettes smoked per day and divided into three groups: daily intake of 20 cigarettes of more; daily intake of less than 20 cigarettes; and nonsmokers (when abstinence from smoking exceeded 10 years).

\section{Colonoscopic procedure}

Bowel preparation included ingesting $4 \mathrm{~L}$ of polyethylene glycol solution. Colonoscopic examinations were performed using two magnification colonoscopes (EC-485 ZW [Fujinon] and EC-3830 FZ [Pentax]), introduced as far as the cecum. Appropriate endoscopic procedures were performed in accordance with the findings, and the morphology, size, number, and location of the lesions were systematically documented.

Then patients underwent chromoscopy of the rectal mucosa, defined as the segment, approximately $15 \mathrm{~cm}$ long, from the dentate line to the rectosigmoid junction. The procedure included the instillation, through a spray catheter, of an average dose of $40 \mathrm{~cm}^{3}$ of $0.2 \%$ methyleneblue, preceded by the instillation of about $10 \mathrm{~cm}^{3}$ of $10 \%$ $\mathrm{N}$-acetylcysteine followed by water wash to remove surface mucus. Under magnification, ACF were defined as lesions in which the crypts were more darkly stained with methylene-blue than normal crypts and had larger diameters, often with oval or slit-like lumens and thicker epithelial linings [23] (Fig. 1). The total number of ACF in rectal mucosa was counted as the procedure was recorded.
The next step involved obtaining biopsy specimens with ACF, at least two per patient. Finally, biopsy specimens were also collected in rectal mucosa with normal endoscopic appearance under magnification (MNEA).

\section{Immunohistochemistry}

Proliferation and apoptosis were evaluated, using immunohistochemistry, in the ACF, MNEA, and carcinomatous tissue obtained from colectomy specimens of surgically resected carcinomas from ten patients (Fig. 2).

Proliferating cells were detected using the MIB-1 antibody (Ki-67 Antigen Kit, Novocastra Laboratories Ltd., Newcastle upon Tyne, UK). Cells were counted as positive if nuclear staining was present.

Apoptotic cells were detected using the M30 antibody (M30 CytoDEATH, Roche Diagnostics GmbH, Penzberg, Germany). Before staining with hematoxylin, each section was incubated with the biotinylated secondary antibody (rabbit antimouse, DakoCytomation Denmark A/S, Glostrup, Denmark) and with streptavid-POD (Roche Diagnostics GmbH, Penzberg, Germany). Cells were counted as positive if cytoplasm staining was present.

For each specimen of ACF, MNEA, or carcinomatous tissue, 1,000 randomly chosen epithelial cells were counted using a light microscope $(\times 400)$. The proliferation index (PI; Ki-67 positive cells/total cells counted) and the apoptotic index (AI; M30 positive cells/total cells counted) were determined.

\section{Statistical analysis}

Statistical comparisons were made, in the case of categorical variables, using the chi-squared test. Mean and standard deviation were used for numerical variables, as well as Bravais-Pearson correlation and the Student $t$ test for the evaluation of differences between means in two
Fig. 1 Endoscopic view of aberrant crypt foci
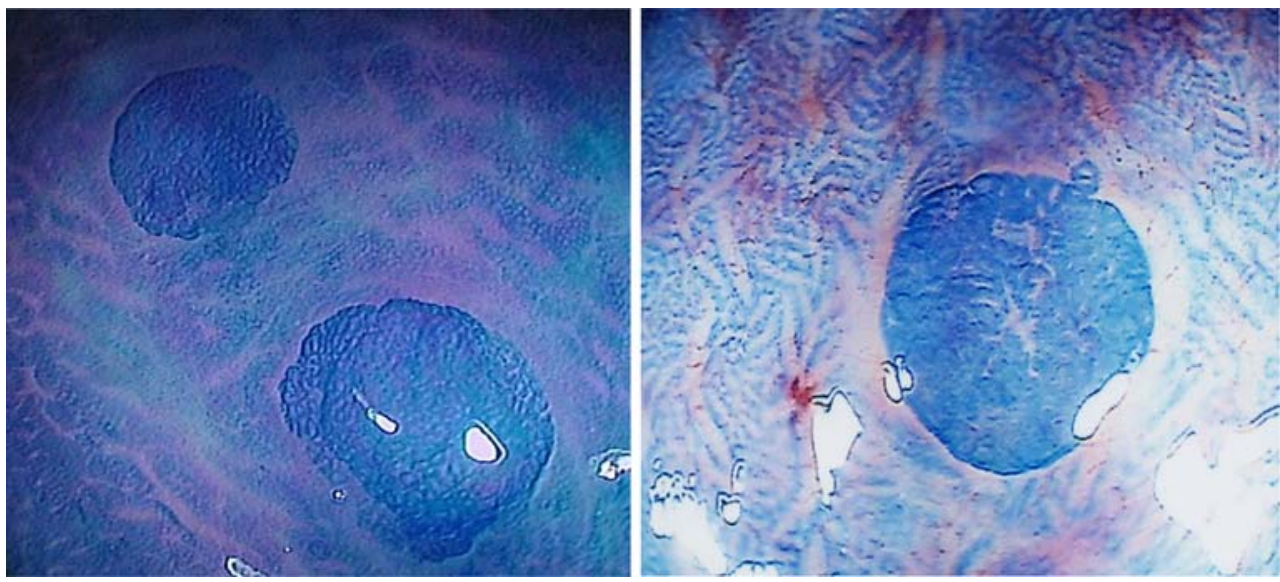

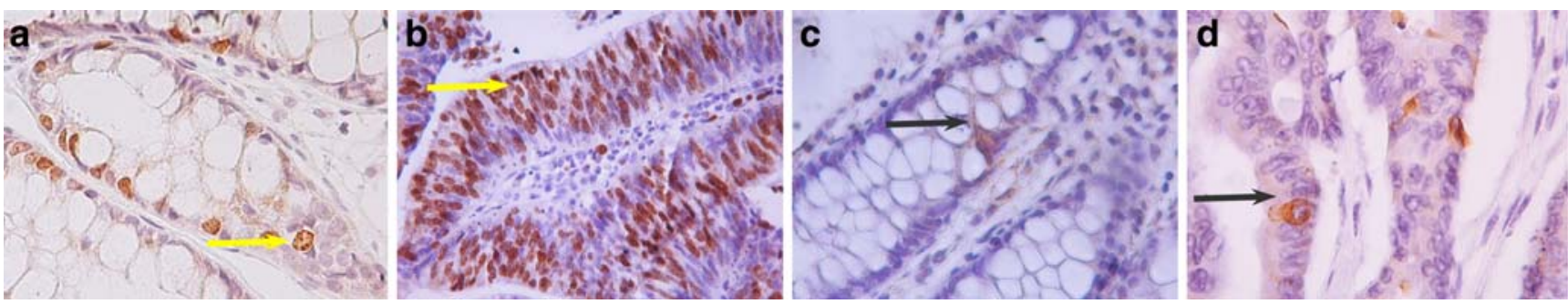

Fig. 2 Proliferation in ACF (a) and in carcinoma (b); apoptosis in ACF (c) and in carcinoma (d); yellow arrow Ki67 expression; black arrow M30 expression ( $\times 40$ magnification)

independent groups and in paired samples. A $p<0.05$ was considered statistically significant. Statistical analysis was performed with statistical software (SPSS version 11.5, SPSS Inc, Chicago, IL, USA).

\section{Results}

The patients were divided into four groups according to the endoscopic findings. Patients with more than one lesion were classified on the basis of the most advanced lesion found.

The group of patients with adenomatous polyps (Group A) included 62 patients (34.1\%), 35 male (56.5\%) and 27 female, with a mean age of 59.9 years and a range of $23-$ 77 years (Table 1).

The group of patients with colorectal carcinoma (Group B) included 55 patients (30.2\%), 39 males (70.9\%) and 16 females, with a mean age of 67.8 years and a range of $45-$ 89 years. The lesion was found in the left colon in 26

Table 1 Patients with adenomatous polyps

\begin{tabular}{lc}
\hline & Patient $[n(\%)]$ \\
\hline Histology & $6(9.7)$ \\
High grade dysplasia & $19(30.6)$ \\
Villous & $14(22.6)$ \\
Tubulovillous & $23(37.1)$ \\
Tubular & \\
Location & $1(1.6)$ \\
Right colon & \\
Left colon & \\
Rectum & $46(74.2)$ \\
Number & $15(24.2)$ \\
More than two & \\
Two & $5(8.1)$ \\
One & $8(12.9)$ \\
Size & $49(79)$ \\
Equal or superior to $1 \mathrm{~cm}$ & \\
Inferior to $1 \mathrm{~cm}$ & $26(41.9)$ \\
\hline
\end{tabular}

${ }^{\text {a }}$ Cecum, ascending colon, hepatic flexure, and transverse colon

${ }^{\mathrm{b}}$ Splenic flexure, descending colon, and sigmoid patients $(47.3 \%)$, in the right colon in $18(32.7 \%)$, and in the rectum in $11(20 \%)$.

The group of patients without colorectal lesions (Group C) included 53 patients (29.1\%), 31 females (58.5\%) and 22 males, with a mean age of 59.8 years and a range of $27-$ 81 years.

The group of patients with nonneoplastic mucosal polyps (Group D) included 12 patients (6.6\%), ten males $(83.3 \%)$ and two females, with a mean age of 53.2 years and a range of 29-70 years. The polyps were hyperplastic in ten patients and juvenile in two.

The patients with carcinoma were predominantly male and significantly older than patients from the other groups. Among drinkers, the number of patients with adenomatous polyps was higher, and in Group B, a larger proportion of patients had a daily intake of over $30 \mathrm{~g}$ of alcohol. On the other hand, more patients in Group D were nondrinkers (Table 2).

The number of rectal ACF was countable in only 178 patients. In the remaining four, difficulties in achieving a good coloration of the rectal mucosa precluded a credible calculation of the number of ACF (Table 3).

The mean number of rectal ACF observed per patient was 7.35 , with a range of $0-55$. Twenty-nine patients (16.2\%) did not show any evidence of rectal ACF (nine from Group A, nine from Group B, seven from Group C, and four from Group D).

Eight hundred fourteen biopsy specimens with ACF were collected - an average of five ACF per patient.

No significant correlation was found between age and the number of ACF.

The AI was significantly lower in the ACF of patients aged 50 years or over $(p=0.035)$. The group classification shows that the AI of the ACF was significantly lower among patients with colorectal neoplasia (Groups A and B) at age 50 or over when compared with younger patients (1.3\% SD 1.6 vs. $2.7 \%$ SD $2.8 ; p=0.01)$. The $\mathrm{AI}$ in the ACF was higher than in the MNEA when the patients were aged under 50 and lower when they were 50 or over. The difference is significant when the group of patients with carcinoma and aged 50 or over is considered (1.1\% SD 1.5 vs $2.1 \%$ SD $2.0 ; p=0.002$ ). The PI was significantly higher in ACF when compared to MNEA among patients aged 
Table 2 The patients' demographic and clinical characteristics

\begin{tabular}{|c|c|c|c|c|c|c|c|}
\hline & & \multicolumn{5}{|c|}{ Groups (number of patients) } & \multirow[t]{2}{*}{$p$ Value } \\
\hline & & A & $\mathrm{B}$ & $\mathrm{C}$ & $\mathrm{D}$ & Total & \\
\hline \multicolumn{2}{|l|}{ Age [Years (SD)] } & $59.9(12.8)$ & $67.8(11.4)$ & $59.8(13.3)$ & $53.2(12.7)$ & $61.8(13.2)$ & $<0.0001$ \\
\hline \multirow[t]{2}{*}{ Gender $[n(\%)]$} & M & $35(56.5)$ & $39(70.9)$ & $22(41.5)$ & $10(83.3)$ & $106(58.2)$ & \multirow[t]{2}{*}{0.005} \\
\hline & $\mathrm{F}$ & $27(43.5)$ & $16(29.1)$ & $31(58.5)$ & $2(16.7)$ & $76(41.8)$ & \\
\hline \multirow[t]{3}{*}{ Tobacco consumption $[n(\%)]$} & 0 & $46(74.2)$ & $46(83.6)$ & $42(79.2)$ & $8(66.7)$ & $142(78.02)$ & \multirow[t]{3}{*}{ NS } \\
\hline & 1 & $9(14.5)$ & $3(5.5)$ & $2(3.8)$ & $1(8.3)$ & $15(8.24)$ & \\
\hline & 2 & $7(11.3)$ & $6(10.9)$ & $9(17)$ & $3(25)$ & $25(13.74)$ & \\
\hline \multirow[t]{3}{*}{ Alcohol consumption $[n(\%)]$} & 0 & $18(29)$ & $19(34.5)$ & $33(62.3)$ & $4(33.3)$ & $74(40.66)$ & \multirow[t]{3}{*}{$<0.0001$} \\
\hline & 1 & $12(19.4)$ & $3(5.5)$ & $10(18.9)$ & 0 & $25(13.74)$ & \\
\hline & 2 & $32(51.6)$ & $33(60)$ & $10(18.9)$ & $8(66.7)$ & $83(45.6)$ & \\
\hline \multirow[t]{3}{*}{ Family history $[n(\%)]$} & 0 & $52(83.9)$ & $49(89.1)$ & $40(75.5)$ & $11(91.7)$ & $152(83.52)$ & \multirow[t]{3}{*}{ NS } \\
\hline & 1 & $3(4.8)$ & $3(5.5)$ & $4(7.5)$ & 0 & $10(5.49)$ & \\
\hline & 2 & $7(11.3)$ & $3(5.5)$ & $9(17)$ & $1(8.3)$ & $20(10.99)$ & \\
\hline \multirow[t]{2}{*}{ Personal history $[n(\%)]$} & 0 & $58(93.5)$ & $55(100)$ & $51(96.2)$ & $12(100)$ & $176(96.7)$ & \multirow[t]{2}{*}{ NS } \\
\hline & 1 & $4(6.5)$ & 0 & $2(3.8)$ & 0 & $6(3.3)$ & \\
\hline \multicolumn{2}{|l|}{ Total } & 62 & 55 & 53 & 12 & 182 & \\
\hline
\end{tabular}

$M$ Male, $F$ female; tobacco consumption: 0 nonsmokers, 1 daily intake of less than 20 cigarettes, 2 daily intake of 20 cigarettes or more; alcohol consumption: 0 nondrinkers, 1 daily intake of less than $30 \mathrm{~g}$ of alcohol, 2 daily intake of over $30 \mathrm{~g}$ of alcohol; family history of colorectal neoplasia: 0 absence, 1 one first-degree relative with colorectal neoplasia diagnosed at age over 60 years or two second-degree relatives with CRC, 2 one first-degree relative with colorectal neoplasia diagnosed at age under 60 years or two first-degree relatives diagnosed with CRC at any age, personal history of colorectal neoplasia: 0 absence, 1 presence; $N S p \geq 0.05, S D$ standard deviation

under $50(p=0.002)$. The group classification shows that this difference is significant $(10.9 \%$ SD 12.4 vs $7.7 \%$ SD $9.1 ; p=0.02)$ among patients with colorectal neoplasia (Groups A and B).

Although not statistically significant, it is noticeable that the IP in the ACF is always higher than in MNEA in both classes of drinkers, as well as in patients that report a family history of colorectal neoplasia and that the IP is higher and the IA lower than in MNEA in patients with a personal history of colorectal neoplasia.

Although the average number of ACF in patients with colorectal neoplasia is higher than in patients without colorectal lesion, the difference is not statistically significant (Table 4).

Table 3 Mean number of ACF, mean IP and IA, in ACF and in MNEA, according to the patients' demographic and clinical characteristics

\begin{tabular}{|c|c|c|c|c|c|c|}
\hline & & \multirow[t]{2}{*}{ Number of ACF (SD) } & \multicolumn{2}{|l|}{ PI (\%) (SD) } & \multicolumn{2}{|l|}{$\mathrm{AI}(\%)(\mathrm{SD})$} \\
\hline & & & $\mathrm{ACF}$ & MNEA & $\mathrm{ACF}$ & MNEA \\
\hline \multirow[t]{2}{*}{ Gender } & M & $7.29(8.62)$ & $9.7(8.5)$ & $8.5(8.3)$ & $1.6(1.9)$ & $2.0(2.4)$ \\
\hline & $\mathrm{F}$ & $7.44(6.04)$ & $10.4(9.4)$ & $11.2(9.0)$ & $1.8(2.2)$ & $1.6(2.0)$ \\
\hline \multirow[t]{2}{*}{ Age } & 0 & $7.64(9.16)$ & $12.6(12.1)^{*}$ & $8.2(8.9)^{*}$ & $2.8(2.8)^{* *}$ & $2.1(2.5)$ \\
\hline & 1 & $7.29(7.29)$ & $9.4(8.0)$ & $10(8.6)$ & $1.5(1.8)^{* *}$ & $1.8(2.2)$ \\
\hline \multirow[t]{3}{*}{ Tobacco consumption } & 0 & $7.42(7.93)$ & $9.2(8.5)$ & $9.3(8.5)$ & $1.7(2.0)$ & $1.9(2.2)$ \\
\hline & 1 & $6.92(6.96)$ & $12.8(11)$ & $13.6(9.7)$ & $1.9(2.4)$ & $1.5(1.8)$ \\
\hline & 2 & $7.16(6.30)$ & $12.4(9.5)$ & $9.6(8.9)$ & $1.5(1.9)$ & $1.7(2.6)$ \\
\hline \multirow[t]{3}{*}{ Alcohol consumption } & 0 & $7.68(8.48)$ & $10.4(8.7)$ & $10.8(8.6)$ & $1.5(1.8)$ & $2.4(2.5)$ \\
\hline & 1 & $8.62(9.96)$ & $11.6(9.1)$ & $10.1(10)$ & $2.5(2.7)$ & $1.4(2.2)$ \\
\hline & 2 & $6.66(5.81)$ & $9.1(9.0)$ & $8.5(8.2)$ & $1.6(2.0)$ & $1.6(1.9)$ \\
\hline \multirow[t]{3}{*}{ Family history } & 0 & $7.41(7.73)$ & $9.4(8.1)$ & $9.6(8.5)$ & $1.7(2.1)$ & $1.9(2.3)$ \\
\hline & 1 & $5.66(2.65)$ & $14.7(13.7)$ & $11.6(11.7)$ & $1.5(1.9)$ & $1.3(1.3)$ \\
\hline & 2 & $7.33(7.75)$ & $11.9(11.5)$ & $9.3(8.6)$ & $1.5(1.6)$ & $1.5(1.8)$ \\
\hline \multirow[t]{2}{*}{ Personal history } & 0 & $4.00(2.44)$ & $9.9(8.9)$ & $9.6(8.5)$ & $1.7(2.1)$ & $1.9(2.3)$ \\
\hline & 1 & $9.15(8.02)$ & $11.2(8.8)$ & $10.8(12.2)$ & $0.7(0.9)$ & $1.4(1.4)$ \\
\hline
\end{tabular}

Age: 0 Under 50 years, 150 years or over

*PI in ACF in patients under 50 years vs PI in MNEA in patients under 50 years $-p$ value below 0.05 ; **AI in ACF in patients under 50 years vs $\mathrm{AI}$ in ACF in patients aged 50 years or over $-p$ value below 0.05 
Table 4 Mean number of ACF, mean IP and IA, in ACF and in MNEA, according to patient group

\begin{tabular}{|c|c|c|c|c|c|}
\hline & \multirow[t]{2}{*}{ Number of ACF (SD) } & \multicolumn{2}{|c|}{ PI (\%) (SD) } & \multicolumn{2}{|l|}{ AI (\%) (SD) } \\
\hline & & $\mathrm{ACF}$ & MNEA & $\mathrm{ACF}$ & MNEA \\
\hline Group A & $8.4(8.3)$ & $8.6(9.3)$ & $8.9(8.8)$ & $1.8(2.1)$ & $1.5(2.3)$ \\
\hline Group B & $7.8(8.8)$ & $10.0(7.9)$ & $9.5(8.7)$ & $1.1(1.5)^{*}, * *$ & $2.0(2.0)^{* *}$ \\
\hline Group C & $5.9(5.0)$ & $11.3(9.4)$ & $11.2(8.8)$ & $2.2(2.4)^{*}$ & $2.0(2.3)$ \\
\hline Group D & $6.1(7.0)$ & $11.4(8.7)$ & $7.5(6.0)$ & $1.7(2.2)$ & $2.1(3.2)$ \\
\hline All patients & $7.3(7.6)$ & $10.0(8.9)$ & $9.7(8.7)$ & $1.7(2.0)$ & $1.9(2.2)$ \\
\hline
\end{tabular}

* $\mathrm{AI}$ in $\mathrm{ACF}$ in group $\mathrm{B}$ vs $\mathrm{AI}$ in $\mathrm{ACF}$ in group $\mathrm{C}-p$ value below $0.05 ; * * \mathrm{AI}$ in $\mathrm{ACF}$ in group $\mathrm{B}$ vs $\mathrm{AI}$ in $\mathrm{MNEA}$ in group $\mathrm{B}-p$ value below 0.05

The AI was significantly lower in the ACF of patients with carcinoma than in patients without colorectal lesion $(p=0.015)$. In those patients, the AI in ACF was significantly lower than in the MNEA $(p=0.002)$. Although not statistically significant, it is clear that when comparing ACF with MNEA, the IP is higher and the IA lower in the ACF.

The rectal location of the neoplasia is associated with a significantly higher mean number of rectal ACF than when the lesion is located elsewhere in the colon $(p=0.01)$. A similar result is found when considering only the patients with adenoma ( $p=0.03)$. When the size of the polyp is $1 \mathrm{~cm}$ or more, the mean number of ACF is significantly superior when compared with patients with smaller lesions $(p=$ 0.02). Although not statistically significant, it is noticeable that the mean number of ACF is higher in patients with more than two polyps and when a high risk of metachronous advanced adenomas is present (Table 5).

The PI in ACF from patients with more than two polyps is significantly higher than in patients with only one or two polyps $(p=0.02)$ and higher than MNEA when the adenoma is located in the rectum $(p=0.03)$. The AI in the ACF is significantly lower when the adenoma is rectal $(p=0.03)$.

In Group D, only the patients with hyperplastic lesions presented rectal ACF. The mean number of rectal ACF in those patients was 7.4.

The mean number of ACF was higher in patients whose ACF presented a higher PI $(p=0.04)$ and a lower AI (NS) than in MNEA (Table 6).

When compared with ACF, carcinomatous tissue showed a significantly higher PI (22\% SD 16.1 vs $10.9 \%$ SD 9; $p=0.02)$ and $\mathrm{AI}(4 \% \mathrm{SD} 2.5$ vs $0.8 \% \mathrm{SD} 0.9 ; p=0.003)$.

The cell kinetics in MNEA, ACF, and CRC is summarized in Fig. 3.

\section{Discussion}

Although the patients included in the study came from a referral center (a possible cause of selection bias), we found, in agreement with published data $[1,36]$, that the patients with carcinoma were older than those with adenoma and that there was a male prevalence when a colorectal neoplasia was present. Concerning alcohol consumption habits, we found that colorectal neoplasia occurrence was more frequent among drinkers and that patients with carcinoma had a higher daily intake of alcohol, which is also in agreement with recent published papers [37-39].

The option to study only rectal ACF, in accordance with studies that applied a similar methodology [19-26], deserves some justification. This decision was founded on investigations in surgical specimens $[40,41]$ and endoscopic studies $[23,42]$ that show that ACF are more frequently detected in the left colon and in the rectum. We also thought that characterizing the ACF with magnification and chromoscopy in the whole colon in a large number of patients would unacceptably prolong the examination time in some cases.

One feasible way to find out whether ACF can be considered precursors of the adenoma carcinoma sequence is to study their connection with known CRC risk factors. Our results show that apoptosis is significantly reduced in the ACF of patients with colorectal neoplasia aged over 50, which suggests that the ability to grow is increased after this age and supports the hypothesis that ACF are involved in the carcinogenic process. In addition, proliferation is significantly higher in ACF when compared with normal mucosa among patients under 50 with colorectal neoplasia, and apoptosis in ACF is lower than in the normal mucosa in patients over 50 with carcinoma. This suggests that under the age of 50, when adenomas prevail, the main process involved in the genesis of the ACF is an increase in proliferation, while after that age, possibly following increased exposure to carcinogenic agents, the reduction of apoptosis may lie at the core of the process.

Apoptosis and proliferation seem to be influenced by other risk factors besides age. Although not statistically significant, it is noticeable that proliferation in the ACF is always higher than in normal mucosa in both classes of drinkers, as well as in patients that report a family history of colorectal neoplasia, while proliferation is higher and apoptosis lower than in normal mucosa in patients with a personal history of colorectal neoplasia. These data, 
Table 5 Mean number of ACF, mean IP and IA, in ACF and in MNEA in patients with neoplastic lesions (Groups A and B) and with adenomas (Group B)

\begin{tabular}{|c|c|c|c|c|c|}
\hline & \multirow[t]{2}{*}{ Number of ACF (SD) } & \multicolumn{2}{|l|}{ PI (\%) (SD) } & \multicolumn{2}{|l|}{ AI (\%) (SD) } \\
\hline & & $\mathrm{ACF}$ & MNEA & $\mathrm{ACF}$ & MNEA \\
\hline \multicolumn{6}{|l|}{ Neoplastic lesions } \\
\hline \multicolumn{6}{|l|}{ Location } \\
\hline Right colon & $6(5.67)^{*}$ & $10.5(8.2)$ & $9.5(9.6)$ & $1.4(1.7)$ & $2.3(2.0)$ \\
\hline Left colon & $7(6.09)^{*}$ & $9.0(8.5)$ & $9.9(8.9)$ & $1.6(2.0)$ & $1.6(2.1)$ \\
\hline Rectum & $12.64(13.62)^{*}$ & $9.0(9.6)^{* *}$ & $6.8(7.7)^{* *}$ & $1.2(1.5)$ & $1.8(2.4)$ \\
\hline \multicolumn{6}{|l|}{ Adenomas } \\
\hline \multicolumn{6}{|l|}{ Location } \\
\hline Left colon & $6.44(5.7)^{* * *}$ & $8.7(8.7)$ & $9.8(8.9)$ & $2.2(2.3)^{* * * *}$ & $1.7(2.4)$ \\
\hline Rectum & $14.35(12.1)^{* * *}$ & $9.1(11.3)$ & $6.9(8.4)$ & $0.8(0.7)^{* * * *}$ & $1.3(2.1)$ \\
\hline \multicolumn{6}{|l|}{ Histology } \\
\hline High grade dysplasia & $9.33(8.59)$ & $6.2(8.5)$ & $3.5(3.1)$ & $2.2(2.9)$ & $0.2(0.3)$ \\
\hline Villous $^{\mathrm{a}}$ & $7.0(9.17)$ & $10.1(10.6)$ & $11.0(10.2)$ & $1.6(1.8)$ & $1.9(2.4)$ \\
\hline Tubular & $10.45(6.59)$ & $7.5(8.0)$ & $7.8(7.4)$ & $1.9(2.2)$ & $1.4(2.3)$ \\
\hline \multicolumn{6}{|l|}{ Number } \\
\hline More than two & $17.25(19.9)$ & $18.1(14.3)^{* * * * *}$ & $18.5(10.1)$ & $2.4(2.0)$ & $3.3(2.5)$ \\
\hline Two & $6.8(8.23)$ & $5.5(6.0)^{* * * * *}$ & $7.0(11.0)$ & $1.3(1.8)$ & $0.2(0.2)$ \\
\hline One & $7.93(6.5)$ & $7.9(8.3)^{* * * * *}$ & $8.0(7.8)$ & $1.8(2.1)$ & $1.5(2.3)$ \\
\hline \multicolumn{6}{|l|}{ Dimension } \\
\hline Equal or superior to $1 \mathrm{~cm}$ & $11.28(10.36)^{* * * * * *}$ & $8.8(10.1)$ & $9.1(10.3)$ & $1.5(1.8)$ & $1.1(1.4)$ \\
\hline Inferior to $1 \mathrm{~cm}$ & $6.27(5.64)^{* * * * * *}$ & $8.6(8.8)$ & $8.7(7.5)$ & $2.1(2.3)$ & $1.9(2.8)$ \\
\hline \multicolumn{6}{|l|}{ Metachronous lesion } \\
\hline High risk ${ }^{\mathrm{b}}$ & $8.53(8.90)$ & $8.8(9.8)$ & $8.5(8.4)$ & $1.8(1.9)$ & $1.5(2.0)$ \\
\hline Reduced risk & $7.88(4.31)$ & $8.1(8.3)$ & $9.9(10.2)$ & $1.8(2.6)$ & $1.6(3.0)$ \\
\hline
\end{tabular}

${ }^{a}$ Includes villous and tubulovillous adenoma

${ }^{\mathrm{b}}$ High risk of metachronous advanced adenomas: more than two adenomas, adenoma equal or larger than $1 \mathrm{~cm}$, villous histology or high-grade dysplasia or family history of CRC [35]

*Number of ACF in patients with rectal neoplastic lesions vs number of ACF in patients with neoplastic lesions in the left and right colon $p$ value below $0.05 ; * * \mathrm{PI}$ in ACF in patients with rectal adenomas vs PI in MNEA in patients with rectal adenomas $-p$ value below 0.05 ; $* * *$ Number of ACF in patients with rectal adenomas vs number of ACF in patients with adenomas in the left colon $-p$ value below 0.05 ; ****AI in ACF in patients with rectal adenomas vs AI in ACF in patients with adenomas in the left colon $-p$ value below 0.05 ; *****PI in ACF in patients with more than two adenomas vs PI in patients with one or two adenomas $-p$ value below 0.05 ; ******Number of ACF in patients with adenomas larger than $1 \mathrm{~cm}$ vs number of ACF in patients with adenomas inferior to $1 \mathrm{~cm}-p$ value below 0.05

suggesting a parallel between cell kinetics in ACF and risk factors for CRC, reinforce the hypothesis that ACF are preneoplastic.

The possible association between ACF and colorectal neoplasia can also be evaluated by their study in the vicinity of neoplastic lesions. As only rectal ACF were considered in this study, their characterization in patients with rectal neoplasia is crucial. The mean number of ACF in patients with rectal neoplasia is significantly higher than in patients with lesions located elsewhere in the colon, a result that is similar when only patients with adenoma are considered. The connection between rectal $\mathrm{ACF}$ and rectal neoplastic lesions is further supported by the significantly higher proliferation index found in the ACF when compared with normal mucosa in patients with rectal lesions and the significantly lower apoptotic index detected in the ACF of these patients. Such findings suggest that the rectal neoplastic lesions might have evolved from one or more of the rectal ACF. This possibility is sustained by the concept of field cancer-

Table 6 Average number of ACF according to AI and PI in ACF vs MNEA

\begin{tabular}{lllll}
\hline & PI (\%) (SD) & & AI (\%) (SD) \\
\cline { 2 - 3 } & ACF $>$ MNEA & ACF $\leq$ MNEA & ACF $<$ MNEA & ACF $\geq$ MNEA \\
\hline Number of ACF(SD) & $10.1(9.4)^{*}$ & $7.6(5.2)^{*}$ & $8.9(8.3)$ & $8.5(6.9)$ \\
\hline
\end{tabular}

*Number of ACF when PI in ACF is higher than in MNEA vs number of ACF when PI in ACF is lower than in MNEA - $p$ value below 0.05 


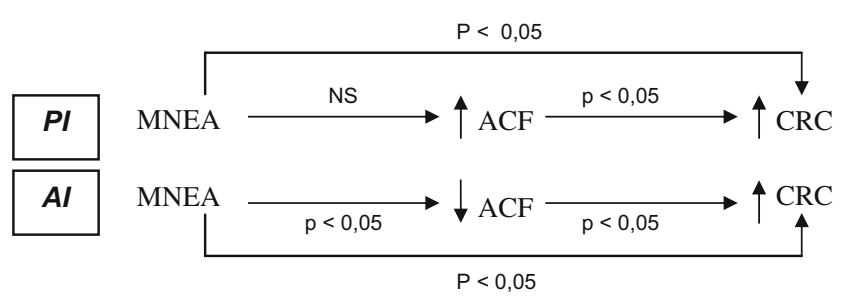

Fig. 3 Cell kinetics in MNEA, ACF, and CRC

ization, put forward to explain the development of multiple primary tumors and locally recurrent cancer, and assuming that multiple areas with similar biological alterations exist in the neighborhood of the carcinomas [43]. These areas, defined for Braakhuis et al. [44] as preneoplastic, are epithelial in nature and, like ACF, of monoclonal origin [45] and may, under certain circumstances, progress to neoplasia.

The evaluation of the rectal ACF according to the pathological findings, namely the presence or absence of colorectal neoplasia, might be relevant because it could help predict the existence of a neoplastic lesion. A comparison of the results of the different series published shows a common trend, in spite of some differences. In fact, Takayama et al. [23], Adler et al. [19], and Yokota et al. [24] found a higher mean number of ACF in patients with benign and malignant colorectal neoplasia than in patients without endoscopic lesions. The paper by Hurlstone et al. [20] on ACF in patients with flat lesions reports similar results, demonstrating that the mean number of ACF increases when patients without lesion, with adenoma, and with carcinoma are considered in succession. In our series, the trend is also toward the presence of a higher mean number of ACF in patients with neoplasia (malignant and benign) than in patients without lesion or with nonneoplastic mucosal polyps.

The link between rectal ACF and colorectal pathology is strengthened when the group of patients with adenoma is stratified according to the characteristics of the polyps. Indeed, the mean number of ACF is significantly higher in patients with bigger polyps. Takayama et al. [23] and Hurlstone et al. [20] found a significant correlation only with the number of polyps. In our series, the mean number of ACF is also higher in patients with more polyps and at high risk of presenting metachronous advanced adenomas, although this is not statistically significant. Proliferation in ACF is higher in patients with bigger polyps and at high risk of metachronous advanced adenomas, the difference being statistically significant in patients with more than two polyps. These results suggest that the characterization of rectal ACF might be useful in stratifying the risk of neoplasia and in postpolypectomy colonoscopic surveillance protocols.

The apoptotic index is related to the presence of colorectal carcinoma. In fact, in patients with this patho- logical finding, apoptosis in the ACF is significantly lower than in patients without lesion and lower than in normal mucosa. As rectal ACF cannot be implicated in the genesis of neoplastic lesions not located in the rectum, one possible explanation is that carcinogenic agents tend to accumulate in the rectum. Indeed, it is possible to speculate that while the carcinogenic agents act along the entire bowel, eventually contributing to the appearance of the neoplastic tissue, they finally accumulate in the rectum, causing the increase in the number of ACF. This parallel, which is in agreement with the results reported by Seike et al. [25], might mean that rectal ACF could be an indicator of a carcinoma elsewhere in the colon.

The way luminal carcinogenic agents reach cells with higher proliferative activity, deeply located in the crypts, is not yet fully understood. Our results, showing that apoptosis in the ACF of patients with carcinoma is significantly lower than in normal mucosa and in patients without colorectal lesion reveals a disturbance in cell kinetics that probably allows cells to remain longer in the upper parts of the crypts, increasing the probability of a luminal carcinogenic agent initiating an advantageous mutation. The results reported by Shpitz et al. [46], according to which the cells in the upper third of ACF maintain a significantly higher proliferative activity than in the same location in normal mucosa, seem to support this hypothesis.

The literature is scarce about ACF in nonneoplastic mucosal polyps, namely of the hyperplastic type. In spite of the small number of patients with this type of polyp considered in our series, it is clear that rectal ACF are also present in patients with hyperplastic polyps.

In our series, when comparing all the ACF analyzed with normal mucosa, proliferation is higher and apoptosis lower (though not statistically significant), a trend that is in accordance with published studies [47, 48]. It might be necessary to increase the sample size or stratify the study according to the crypt compartments to achieve statistical significance.

As expected, a higher proliferation index in the ACF than in the normal mucosa is associated with a significantly higher mean number of ACF. A lower apoptotic index in the ACF is also linked with a higher mean number of ACF. This connection between cell kinetics and the mean number of ACF confers credibility on the endoscopic technique of detection and counting of rectal ACF.

It is a known fact that proliferation is higher in carcinomatous tissue than in normal mucosa or in ACF [48]. With regard to apoptosis, contradictory results are found when carcinomas and adenomas are compared; some papers report a decrease [49, 50] and some an increase [51]. Our study shows that both proliferation and apoptosis are significantly higher in carcinoma than in ACF. The increased apoptosis could be the result of the lack of 
nutrients and oxygen caused by the fast growth of the lesion [52]. The apparent discrepancy between the existence of malignant tissue and a rise in apoptosis may be explained by a proportionally lesser increase when compared with proliferation [51].

In our series, different results were obtained when we compared proliferation and apoptosis in ACF with normal mucosa and carcinoma. In fact, while apoptosis significantly decreases and proliferation increases in ACF compared to normal mucosa, apoptosis and proliferation are significantly higher in carcinoma versus ACF. Given these data, we can speculate that the main disturbance in cell kinetics involved in the progression from normal mucosa to ACF is a decrease in apoptosis, while the admitted progression of the ACF to CRC could be the result of a marked increase in proliferation accompanied by a proportionately inferior increase in apoptosis unable to prevent the tumor growth. The reports of a progressive increase in proliferative activity accompanied, in the final phase of the carcinogenesis, by a significant increase in apoptosis $[48,51]$ is in agreement with our results, reinforcing the hypothesis that ACF are part of the carcinogenic process.

In conclusion, the results of our study, by showing that the number and cell kinetics of the ACF vary according with the vicinity of neoplastic lesion and with known risk factors for colorectal cancer, namely age, support the assumption that ACF are preneoplastic. Rectal ACF characterization may be helpful in predicting the presence of a colorectal neoplasia because there is a tendency towards a higher mean number of ACF in patients with neoplasia; patients with polyps bigger than $1 \mathrm{~cm}$ present significantly more rectal ACF, and in patients with carcinoma, irrespective of the location of the lesion, the rectal ACF apoptotic index is lower than in the normal mucosa and in patients without colorectal lesions.

\section{Potential competing interests: none}

Financial support: this study was supported by "FCT-Fundação para a Ciência e a Tecnologia" (POCTI/0060/2003)

\section{References}

1. Parkin DM, Bray F, Ferlay J et al (2005) Global cancer statistics, 2002. CA Cancer J Clin 55:74-108

2. Rex DK, Johnson DA, Lieberman DA et al (2000) Colorectal cancer prevention 2000: screening recommendations of the American College of Gastroenterology. American College of Gastroenterology. Am J Gastroenterol 95:868-877

3. Vogelstein B, Fearon ER, Hamilton SR et al (1988) Genetic alterations during colorectal-tumor development. N Engl J Med 319:525-532
4. Bostick RM, Fosdick L, Grandits GA et al (1997) Colorectal epithelial cell proliferative kinetics and risk factors for colon cancer in sporadic adenoma patients. Cancer Epidemiol Biomarkers Prev 6:1011-1019

5. Roncucci L, Scalmati A, Ponz de Leon M (1991) Pattern of cell kinetics in colorectal mucosa of patients with different types of adenomatous polyps of the large bowel. Cancer 68:873-878

6. Sandler RS, Baron JA, Tosteson TD et al (2000) Rectal mucosal proliferation and risk of colorectal adenomas: results from a randomized controlled trial. Cancer Epidemiol Biomarkers Prev 9:653-656

7. Scalmati A, Roncucci L, Ghidini G et al (1990) Epithelial cell kinetics in the remaining colorectal mucosa after surgery for cancer of the large bowel. Cancer Res 50:7937-7941

8. Freitas D, Goulão MH, Camacho E et al (2002) Clinical relevance of proliferation biomarkers and p53 expression in rectal mucosa and sporadic colonic adenomas: a prospective study. Hepatogastroenterology 49:1269-1274

9. Anti M, Armuzzi A, Morini S et al (2001) Severe imbalance of cell proliferation and apoptosis in the left colon and in the rectosigmoid tract in subjects with a history of large adenomas. Gut 48:238-246

10. Martin C, Connelly A, Keku TO et al (2002) Nonsteroidal antiinflammatory drugs, apoptosis, and colorectal adenomas. Gastroenterology 123:1770-1777

11. Bernstein C, Bernstein H, Garewal H et al (1999) A bile acidinduced apoptosis assay for colon cancer risk and associated quality control studies. Cancer Res 59:2353-2357

12. Bird RP (1987) Observation and quantification of aberrant crypts in the murine colon treated with a colon carcinogen: preliminary findings. Cancer Lett 37:147-151

13. Boivin GP, Washington K, Yang K et al (2003) Pathology of mouse models of intestinal cancer: consensus report and recommendations. Gastroenterology 124:762-777

14. Bird RP, McLellan EA, Bruce WR (1989) Aberrant crypts, putative precancerous lesions, in the study of the role of diet in the aetiology of colon cancer. Cancer Surv 8:189-200

15. McLellan EA, Medline A, Bird RP (1991) Dose response and proliferative characteristics of aberrant crypt foci: putative preneoplastic lesions in rat colon. Carcinogenesis 12:2093-2098

16. Mereto E, Frencia L, Ghia M (1994) Effect of aspirin on incidence and growth of aberrant crypt foci induced in the rat colon by 1,2dimethylhydrazine. Cancer Lett 76:5-9

17. Shpitz B, Bomstein Y, Kariv N et al (1998) Chemopreventive effect of aspirin on growth of aberrant crypt foci in rats. Int $\mathrm{J}$ Colorectal Dis 13:169-172

18. Wargovich MJ, Chen CD, Harris C et al (1995) Inhibition of aberrant crypt growth by non-steroidal anti-inflammatory agents and differentiation agents in the rat colon. Int J Cancer 60:515-519

19. Adler DG, Gostout CJ, Sorbi D et al (2002) Endoscopic identification and quantification of aberrant crypt foci in the human colon. Gastrointest Endosc 56:657-662

20. Hurlstone DP, Karajeh M, Sanders DS et al (2005) Rectal aberrant crypt foci identified using high-magnification-chromoscopic colonoscopy: biomarkers for flat and depressed neoplasia. Am J Gastroenterol 100:1283-1289

21. Moxon D, Raza M, Kenney R et al (2005) Relationship of aging and tobacco use with the development of aberrant crypt foci in a predominantly African-American population. Clin Gastroenterol Hepatol 3:271-278

22. Rudolph RE, Dominitz JA, Lampe JW et al (2005) Risk factors for colorectal cancer in relation to number and size of aberrant crypt foci in humans. Cancer Epidemiol Biomarkers Prev 14:605-608

23. Takayama T, Katsuki S, Takahashi Y et al (1998) Aberrant crypt foci of the colon as precursors of adenoma and cancer. N Engl J Med 339:1277-1284 
24. Yokota T, Sugano K, Kondo H et al (1997) Detection of aberrant crypt foci by magnifying colonoscopy. Gastrointest Endosc 46:61-65 Jul

25. Seike K, Koda K, Oda K et al (2006) Assessment of rectal aberrant crypt foci by standard chromoscopy and its predictive value goes colonic advanced neoplasms. Am J Gastroenterol 101:1362-1369

26. Stevens RG, Swede H, Heinen CD et al (2007) Aberrant crypt foci in patients with the positive family history of sporadic colorectal cancer. Cancer Lett 248:262-268

27. Konstantakos AK, Siu IM, Pretlow TG et al (1996) Human aberrant crypt foci with carcinoma in situ from a patient with sporadic colon cancer. Gastroenterology 111:772-777

28. Otori K, Konishi M, Sugiyama K et al (1998) Infrequent somatic mutation of the adenomatous polyposis coli gene in aberrant crypt foci of human colon tissue. Cancer 83:896-900

29. Takayama T, Ohi M, Hayashi T et al (2001) Analysis of K-ras, APC, and beta-catenin in aberrant crypt foci in sporadic adenoma, cancer, and familial adenomatous polyposis. Gastroenterology 121:599-611

30. Pedroni M, Sala E, Scarselli A et al (2001) Microsatellite instability and mismatch-repair protein expression in hereditary and sporadic colorectal carcinogenesis. Cancer Res 61:896-899

31. Winawer S, Fletcher R, Rex D et al (2003) Colorectal cancer screening and surveillance: clinical guidelines and rationaleUpdate based on new evidence. Gastroenterology 124:544-560

32. Bagnardi V, Blangiardo M, La Vecchia C et al (2001) A metaanalysis of alcohol drinking and cancer risk. $\mathrm{Br} \mathrm{J}$ Cancer 85:1700-1705

33. Cho E, Smith-Warner SA, Ritz J et al (2004) Alcohol intake and colorectal cancer: a pooled analysis of 8 cohort studies. Ann Intern Med 140:603-613

34. Giovannucci E (2001) An updated review of the epidemiological evidence that cigarette smoking increases risk of colorectal cancer. Cancer Epidemiol Biomarkers Prev 10:725-731

35. Bond JH (2000) Polyp guideline: diagnosis, treatment, and surveillance for patients with colorectal polyps. Practice Parameters Committee of the American College of Gastroenterology. Am J Gastroenterol 95:3053-3063

36. Lieberman DA, Weiss DG, Bond JH et al (2000) Use of colonoscopy to screen asymptomatic adults for colorectal cancer. Veterans Affairs Cooperative Study Group 380. N Engl J Med 343:162-168

37. Anderson JC, Alpern Z, Sethi G et al (2005) Prevalence and risk of colorectal neoplasia in consumers of alcohol in a screening population. Am J Gastroenterol 100:2049-2055

38. Bongaerts BW, of Goeij AF, of Vogel S et al (2007) Alcohol consumption and distinct molecular pathways to colorectal cancer. Br J Nutr 97:430-434
39. Gondal G, Grotmol T, Hofstad B et al (2005) Lifestyle-related risk factors and chemoprevention for colorectal neoplasia: experience from the large-scale NORCCAP screening trial. Eur J Cancer Prev 14:373-379

40. Nucci MR, Robinson CR, Longo P et al (1997) Phenotypic and genotypic characteristics of aberrant crypt foci in human colorectal mucosa. Hum Pathol 28:1396-1407

41. Roncucci L, Modica S, Pedroni M et al (1998) Aberrant crypt foci in patients with colorectal cancer. Br J Cancer 77:2343-2348

42. Takayama T, Ohi M, Hayashi T et al (2001) Analysis of K-ras, APC, and beta-catenin in aberrant crypt foci in sporadic adenoma, cancer, and familial adenomatous polyposis. Gastroenterology 121:599-611

43. Jothy S, Slesak B, Harłozińska A et al (1996) Field effect of human colon carcinoma on normal mucosa: relevance of carcinoembryonic antigen expression. Tumour Biol 17:58-64

44. Braakhuis BJ, Tabor MP, Kummer JA et al (2003) A genetic explanation of Slaughter's concept of field cancerization: evidence and clinical implications. Cancer Res 63:1727-1730

45. Siu IM, Robinson DR, Schwartz S et al (1999) The identification of monoclonality in human aberrant crypt foci. Cancer Res 59:63-66

46. Shpitz B, Bomstein Y, Mekori Y et al (1997) Proliferating cell nuclear antigen as a marker of cell kinetics in aberrant crypt foci, hyperplastic polyps, adenomas, and adenocarcinomas of the human colon. Am J Surg 174:425-430

47. Nobuoka A, Takayama T, Miyanishi K et al (2004) Glutathione-Stransferase P1-1 protects aberrant crypt foci from apoptosis induced by deoxycholic acid. Gastroenterology 127:428-443

48. Shpitz B, Bomstein Y, Mekori Y et al (1997) Proliferating cell nuclear antigen as a marker of cell kinetics in aberrant crypt foci, hyperplastic polyps, adenomas, and adenocarcinomas of the human colon. Am J Surg 174:425-430

49. Aotake T, Lu CD, Chiba Y et al (1999) Changes of angiogenesis and tumor cell apoptosis during colorectal carcinogenesis. Clin Cancer Res 5:135-142

50. Tsujitani S, Shirai H, Tatebe S et al (1996) Apoptotic cell death and its relationship to carcinogenesis in colorectal carcinoma. Cancer 77:1711-1716

51. Hao X, Du M, Bishop AE et al (1998) Imbalance between proliferation and apoptosis in the development of colorectal carcinoma. Virchows Arch 433:523-527

52. Koornstra JJ, de Jong S, Hollema H et al (2003) Changes in apoptosis during the development of colorectal cancer: a systematic review of the literature. Crit Rev Oncol Hematol $45: 37-53$ 